\title{
Prenatal NMDA Receptor Antagonism Impaired Proliferation of Neuronal Progenitor, Leading to Fewer Glutamatergic Neurons in the Prefrontal Cortex
}

\author{
Kazuya Toriumi ${ }^{1,2}$, Akihiro Mouri, ${ }^{1,3}$, Shiho Narusawa', Yuki Aoyama', Natsumi Ikawa', Lingling Lu',2, \\ Taku Nagai ${ }^{3}$, Takayoshi Mamiya ${ }^{1,2}$, Hyoung-Chun Kim ${ }^{4}$ and Toshitaka Nabeshima*,, \\ 'Department of Chemical Pharmacology, Graduate School of Pharmaceutical Sciences, Meijo University, Nagoya, Japan; ${ }^{2}$ The Academic \\ Frontier Project for Private University, Comparative Cognitive Science Institutes, Meijo University, Nagoya, Japan; ${ }^{3}$ Department of \\ Neuropsychopharmacology and Hospital Pharmacy, Nagoya University Graduate School of Medicine, Nagoya, Japan; ${ }^{4}$ Neurotoxicology Program, \\ Department of Pharmacy, College of Pharmacy, Kangwon National University, Korea Institute of Drug Abuse, Chunchon, South Korea
}

\begin{abstract}
N-methyl-D-aspartate (NMDA) receptor is a glutamate receptor which has an important role on mammalian brain development. We have reported that prenatal treatment with phencyclidine (PCP), a NMDA receptor antagonist, induces long-lasting behavioral deficits and neurochemical changes. However, the mechanism by which the prenatal antagonism of NMDA receptor affects neurodevelopment, resulting in behavioral deficits, has remained unclear. Here, we report that prenatal NMDA receptor antagonism impaired the proliferation of neuronal progenitors, leading to a decrease in the progenitor pool in the ventricular and the subventricular zone. Furthermore, using a PCR array focused on neurogenesis and neuronal stem cells, we evaluated changes in gene expression causing the impairment of neuronal progenitor proliferation and found aberrant gene expression, such as Notch2 and Ntn I, in prenatal PCP-treated mice. Consequently, the density of glutamatergic neurons in the prefrontal cortex was decreased, probably resulting in glutamatergic hypofunction. Prenatal PCP-treated mice displayed behavioral deficits in cognitive memory and sensorimotor gating until adulthood. These findings suggest that NMDA receptors regulate the proliferation and maturation of progenitor cells for glutamatergic neuron during neurodevelopment, probably via the regulation of gene expression.

Neuropsychopharmacology (2012) 37, 1387-1396; doi:10.1038/npp.201 I.324; published online I8 January 2012
\end{abstract}

Keywords: NMDA receptor; phencyclidine; glutamatergic neuron; neurogenesis; neuronal progenitor; schizophrenia

\section{INTRODUCTION}

A variety of amino acids and corresponding receptors, which mediate fast synaptic transmission in the central nervous system (CNS), are present before birth where they are considered to have a role in the morphogenesis of the CNS at different stages, including proliferation, migration, and differentiation. Glutamate is a major amino acid, acting on at least five types of receptors. Of the ionotropic receptors, the $N$-methyl-D-aspartate (NMDA) receptor has critical roles in neurogenesis. The expression of NMDA receptors has been described in certain cell lines and in precursor cells of the developing and adult CNS (Asahi et al, 1998). Functional NMDA receptors have been found in radial glia cells (López et al, 1997), which act as neuronal

\footnotetext{
*Correspondence: Professor T Nabeshima, Department of Chemical Pharmacology, Graduate School of Pharmaceutical Sciences, Meijo University, 150 Yagotoyama, Tenpaku-ku, Nagoya, 468-8503, Japan, Tel: +8I 52839 2735, Fax: + 8152839 2738,

E-mail: tnabeshi@meijo-u.ac.jp

Received 8 June 2011; revised 3 December 2011; accepted 5 December 201 I
}

progenitors during cortical development (Heins et al, 2002). Glutamate has been also reported to regulate the proliferation of progenitor cells derived from the perinatal subventricular zone (SVZ) through both ionotropic and metabotropic receptors, including NMDA receptors (Brazel et al, 2005). Moreover, the growth of primary cultures of embryonic hippocampal progenitor cells in proliferative conditions was associated with low levels of NMDA currents (Sah et al, 1997).

Phencyclidine (PCP) is a noncompetitive NMDA receptor antagonist. The acute and chronic administration of PCP can induce schizophrenia-like symptoms in both humans and rodents, findings that have greatly contributed to a hypoglutamatergic hypothesis of schizophrenia. Additionally, the administration of PCP during development in rodents induces schizophrenia-like impairments in sensorimotor gating and spatial learning later in life (Wang et al, 2001). Previously, we reported that prenatal PCP treatment caused an impairment of cognitive memory, sensitization to PCP, and sensorimotor gating deficits (Lu et al, 2010, 2011). Based on the neurodevelopmental hypothesis of schizophrenia, prenatal PCP-treated animals would be a better 
pharmacological model of schizophrenia than adult PCPtreated animals. However, the mechanism by which prenatal NMDA antagonism impairs neurodevelopment, resulting in long-lasting schizophrenia-like deficits, has remained unclear.

In this study, we found that prenatal PCP treatment disturbed gene expression in neuronal progenitors and consequently impaired cell proliferation, causing the density of glutamatergic neurons to decrease in the prefrontal cortex (PFC), an area critical in schizophrenia patients, resulting in schizophrenia-like deficits until adulthood.

\section{SUBJECTS AND METHODS}

\section{Animals}

Breeder and host ICR wild-type mice were obtained from SLC Japan (Shizuoka, Japan). Noon on the day a vaginal plug was detected embryonic day (E) 0.5. E19.5 was defined as postnatal day (P) 1. After weaning on P28, pups given the same prenatal treatment were mixed by gender and randomly assigned to groups for behavioral tests on P28 and P56. Two or three litters were used in each group, and the test was repeated more than three times to reduce the influence of litters. Moreover, a balanced number of males and females were used in each experiment, because there were no significant differences between genders in this study.

\section{PCP and 5-Bromo-2-Deoxyuridine (BrdU) Administration}

PCP hydrochloride was synthesized according to the method of Maddox et al (1965) and checked for purity through measurements of its melting point and ultraviolet spectrum. PCP dissolved in saline (SAL) was administered (10 mg/kg/day, s.c.) to pregnant dams from E6.5 to E18.5.

To label neural progenitors cycling in S-phase in E12.5, E13.5, E14.5, or E15.5 embryos, pregnant mice were injected intraperitoneally with $50 \mathrm{mg} / \mathrm{kg}$ body weight of $\mathrm{BrdU}$ (Sigma) in SAL $3 \mathrm{~h}$ after the PCP treatment.

\section{Novel Object Recognition Test}

The novel object recognition test was carried out as described previously (Mouri et al, 2007) with minor modifications. The test procedure consisted of three sessions: habituation, training, and retention. Each mouse was individually habituated to the box, with $10 \mathrm{~min}$ of exploration in the absence of objects each day for 3 consecutive days (days 1-3) (habituation session). On day 4, each animal was allowed to explore for $10 \mathrm{~min}$ in the box, in which two novel objects were placed symmetrically. The time spent exploring each object was recorded (training session). The objects were different in shape and color, but similar in size. Animals were considered to be exploring an object when their heads were facing it or when they were sniffing it at a distance of $<2 \mathrm{~cm}$ and/or touching it with their nose. After the training session, mice were immediately returned to their home cages. On day 5, the animals were placed back into the same box with one of the familiar objects used in the training session and one novel object.
Animals were allowed to explore freely for $10 \mathrm{~min}$ and the time spent exploring each object was recorded (retention session). An exploratory preference, the ratio of time spent exploring either of the two objects (training session) or the novel object (retention session) over the total amount of time spent exploring both objects, was used to assess recognition memory.

\section{Forced Swimming Test}

Each mouse was placed in a transparent glass cylinder (20 cm high, $15 \mathrm{~cm}$ in diameter), which contained water at $22-23{ }^{\circ} \mathrm{C}$ to a depth of $15 \mathrm{~cm}$, and was forced to swim for $4 \mathrm{~min}$. We measured the duration of swimming every minute with an infrared detector, SCANET MV-10 AQ (Melquest, Japan), and then calculated 'immobility time' using the score of swimming duration as follows: total time (s)-swimming time (s)= immobility time (s). Total immobility time was calculated for $3 \mathrm{~min}$ except the first $1 \mathrm{~min}$.

\section{Prepulse Inhibition (PPI) Test}

PPI of the acoustic startle response was measured using an SR-LAB System (San Diego Instruments). The stimulus consisted of a $20-\mathrm{ms}$ prepulse, a $100-\mathrm{ms}$ delay, and then a $40-\mathrm{ms}$ startle pulse. The intensity of the prepulse was 4,8 , or $16-\mathrm{dB}$ above the 70- $\mathrm{dB}$ background noise. The amount of PPI was calculated as a percentage of the $120-\mathrm{dB}$ acoustic startle response: $100-(($ startle reactivity on prepulse + startle pulse)/startle reactivity on startle pulse) $\times 100$.

\section{Preparation of Brain Slice and Staining}

Mice were anesthetized intraperitoneally with ethyl carbamate at a dose of $1.2 \mathrm{~g} / \mathrm{kg}$ and perfused transcardially with ice-cold phosphate-buffered SAL (PBS), followed by $4 \%$ paraformaldehyde (PFA) in PBS. The brains were removed, postfixed in the same fixative overnight, and then soaked in $30 \%(\mathrm{w} / \mathrm{v})$ sucrose in PBS. Coronal sections $20-40 \mu \mathrm{m}$ thick were cut with a cryostat (Micro-edge Instruments, Japan). Neonatal brains were fixed overnight in $4 \%$ PFA, cryoprotected in $30 \%$ sucrose, and cut at $8 \mu \mathrm{m}$. For counting the number and measuring the size of neurons, cresyl violet staining was performed and neurons with a visible nucleus and apparent outline of the entire cell were counted in a standardized area in the prelimbic region using a computerbased image analysis system, WinROOF (Mitani, Japan). Using these measurements, the upper and lower areas distinguished by the size of neurons, were calculated in a standardized area in layers II/III and IV-VI of the prelimbic region, respectively. Images were acquired with a light microscope (Biorevo BZ-9000; KEYENCE, Axioskop2 plus; Carl Zeiss). For immunohistochemistry, the brain slices were incubated with primary antibodies such as antiphosphate-activated glutaminase (PAG) (1:500; Aviva Systems Biology), anti-NeuN (1:1000; Chemicon), antiBrdU (1:10; from BrdU Labeling and Detection Kit II, Roche), anti-Pax6 (1:1000; Abcam), anti-proliferation cell nuclear antigen (PCNA) (1:5000; DAKO), and anti-Tbr2 ( $1: 500 ;$ Abcam) antibody. Fluorescently conjugated secondary antibodies (Alexa 488 and 546; Molecular Probes) 
were also used. Images were acquired with LSM510 meta (Carl Zeiss), and then the immuno-positive cells in the prelimbic region were counted by WinROOF.

\section{Laser Capture Microdissection (LCM)}

We collected samples of the proliferative ventricular zone (VZ) and SVZ from E13.5 mice by using LCM. Embryos were removed from dams $3 \mathrm{~h}$ after the administration of PCP and decapitated; their heads were frozen in liquid nitrogen and stored at $-80^{\circ} \mathrm{C}$. The frozen heads were sectioned on a cryostat at a thickness of $20 \mu \mathrm{m}$ in the coronal plane. The sections were mounted on MembraneSlide 1.0 PEN (Carl Zeiss), dried at room temperature for $1 \mathrm{~min}$, and immediately fixed in methanol for $3 \mathrm{~min}$. They were counterstained using a LCM staining kit (Ambion) and viewed under a PALM-MBC MicroBeam System (PALM Microlaser Technologies GmbH, Germany). The VZ and SVZ areas in the developing cerebral cortex were identified and dissected. Immediately after the dissection, the sample was picked up on adhesive lids of $500 \mu$ l Adhesive Caps (PALM Microlaser Technologies $\mathrm{GmbH}$ ). The cut out samples were collected from both hemispheres in 6-8 sections of one brain.

\section{PCR Array Focused on Neurogenesis and Neural Stem Cells}

RNA from the LCM samples was extracted with RNAqueous-Micro (Ambion) according to the manufacturer's instructions. Reverse transcription was carried out using an $\mathrm{RT}^{2}$ PCR Array First Strand Kit (SA Bioscience) following the manufacturer's instructions. A total of $1 \mu \mathrm{g}$ of RNA per sample was used. An aliquot of the diluted cDNA template was stored at $-30{ }^{\circ} \mathrm{C}$ and further used in a reverse transcription-polymerase chain reaction (RT-PCR) with an ABI 7500, RT $^{2}$ Real-Time SYBR Green PCR Master Mix, and Mouse Neurogenesis and Neural Stem Cells RT ${ }^{2}$ Profiler PCR Array (SA Bioscience), according to the manufacturer's directions. The threshold cycle $(\mathrm{Ct})$ for each well was calculated using the instrument's software. To ensure that the baseline and threshold were the same across all PCR arrays run in the same analysis, internal controls provided by the manufacturer were used. Data analysis was run by the $\Delta \Delta$ Ct method (Pfaffl, 2001).

Furthermore, this PCR array system contains a genomic DNA control, a reverse transcription control, and a positive PCR control. The genomic DNA control was to specifically detect non-transcribed genomic DNA contamination with a high level of sensitivity. The reverse transcription control tests the efficiency of the $\mathrm{RT}^{2}$ First Strand Kit reaction with a primer set detecting the template synthesized from the kit's built-in external RNA control. The positive PCR control tests the efficiency of the polymerase chain reaction itself using a pre-dispensed artificial DNA sequence and the primer set that detects it. We confirmed that these controls were normal, and then analyzed the data obtained.

\section{Statistical Analysis}

All data were expressed as the mean \pm SEM. The statistical significance of differences between two groups was determined with Student's $t$-test. The significance of differences in FST and PPI was determined using a twoway analysis of variance (ANOVA) with repeated measures, followed by Student's $t$-test. $p<0.05$ was regarded as statistically significant.

\section{RESULTS}

\section{Prenatal PCP-Treatment Reduced Body Weight in Newborn Pups}

To evaluate effects of prenatal NMDA antagonism on neurodevelopment, we administered PCP at a dose of $10 \mathrm{mg} / \mathrm{kg} /$ day to pregnant dams from E6.5 to E18.5. The body weight of PCP-treated dams decreased day by day, and significantly on E18.5 compared with SAL-treated mice (Figure 1a). Furthermore, PCP treatment also significantly reduced the body weight of newborn pups on P1 compared with SAL-treated mice without any change in the number of pups (Figures $1 \mathrm{~b}$ and $\mathrm{c}$ ). However, after birth, the difference decreased gradually with growth, so that there was no change on P28 and 56 (Figure 1d). These results suggest that prenatal PCP-treatment induced some developmental deficits without embryonic lethality.
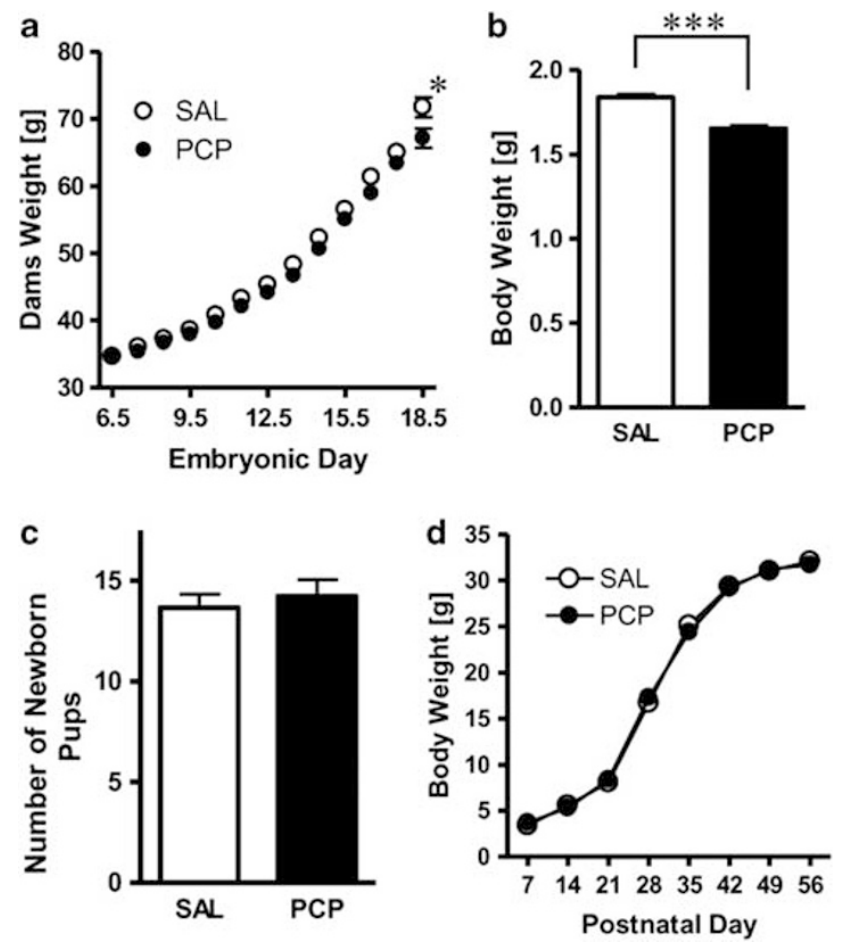

Figure I Prenatal PCP-treatment reduced body weight in newborn pups. Mice were administrated SAL or PCP from E6.5 to El 8.5. (a) Changes in the body weight of the dams are shown (two-way ANOVA with repeated measures: $F_{\text {Interaction(12, 132) }}=3.01 ; p<0.05, F_{\text {Time }(12,132)}=1190$;

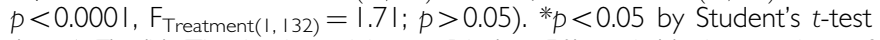
$(n=6-7)$. (b) The body weight on $\mathrm{PI}(n=50)$ and (c) the number of newborn pups $(n=6-9)$ are shown. $* * * * 00.00$ I by Student's $t$-test. (d) Changes in the body weight of the pups after birth are shown $(n=24-28)$ (two-way ANOVA with repeated measures: $F_{\text {Interaction }(7,400)}=0.27$; $\left.p>0.05, F_{\text {Time }(7,400)}=78 I ; p<0.000 I, F_{\text {Treatment }(I, 400)}=0.01 ; p>0.05\right)$. Values are means \pm SEMs. 
Prenatal PCP-Treatment Induced Behavioral Deficits in Adulthood

To investigate the effect of prenatal NMDA receptorantagonism on behavior in adulthood, we administered PCP at a dose of $10 \mathrm{mg} / \mathrm{kg} /$ day to pregnant dams from E6.5 to E18.5, and tested the behavioral performance of their pups from P28 (Figure 2a). In novel object recognition tests on P56 to investigate the effects on recognition memory, PCP-treated mice showed changes in exploratory preference during the retention session compared with SAL-treated mice, although there was no change in exploratory time (Figures $2 \mathrm{~b}$ and $\mathrm{c}$ ). In the forced swimming test, the PCPtreated mice showed an increase in immobility time in comparison with SAL-treated mice, suggesting behavioral deficits like negative symptoms of schizophrenia (Figures $2 \mathrm{~d}$ and e). These behavioral deficits were expressed from P28 (Supplementary Figure S1). Furthermore, in the test to evaluate sensory motor gating function, which is frequently impaired in schizophrenia patients, prenatal PCP treatments significantly reduced PPI compared with SAL (Figures $2 \mathrm{f}$ and $\mathrm{g}$ ). These results indicated that prenatal PCP-treatments induced schizophrenia-like behavioral deficits in adulthood, similar to our previous report using PCP at a high dose of $20 \mathrm{mg} / \mathrm{kg}$ (Lu et al, 2010).

\section{Prenatal PCP-Treatment Reduced the Density of Glutamatergic Neurons in PFC}

To reveal the cause of these behavioral deficits, we investigated the effect of PCP-treatments on neurodevelopment in the PFC, an area critical to schizophrenia. First, we examined morphological and numerical changes in the neurons on P56 by cresyl violet staining (Figure 3a). No difference in the size of neurons in the upper (II/III) or lower (IV-VI) layer of the PFC was observed regardless of PCP treatment (Figure $3 \mathrm{~b}$ ). However, a decrease in the density of neurons was revealed in both layers of the PFC in PCP-treated mice compared with SAL-treated mice (Figure $3 \mathrm{c}$ ).

It has been reported that hypoglutamatergic function in the PFC was found in patients with schizophrenia and an animal model treated with PCP chronically, which shows abnormal behavior (Lewis et al, 2003; Murai et al, 2007). Thus, we checked the density of glutamatergic neurons in the PFC of PCP-treated mice by immunohistochemical analysis. PAG was used as a glutamatergic neuronal marker, because it is detected only in pyramidal neurons, not in GABA-immunoreactive neurons or in nonpyramidal neurons (Kaneko and Mizuno, 1994). The result showed that number of glutamatergic neurons were reduced significantly in PCP-treated mince compared with SAL-treated mice (Figures 3d and e). These results indicated that prenatal NMDA antagonism impaired glutamatergic neurogenesis.

\section{NMDA Receptor Antagonism Impaired Neurogenesis but not the Neural Migration}

Glutamatergic neurons in the PFC are produced in the VZ and SVZ, and then migrate radially to the correct site, resulting in the formation of a layered structure with an inside-out pattern (Dehay and Kennedy, 2007). It has been a

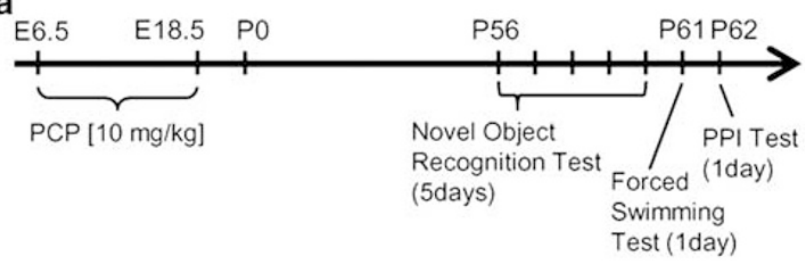

b
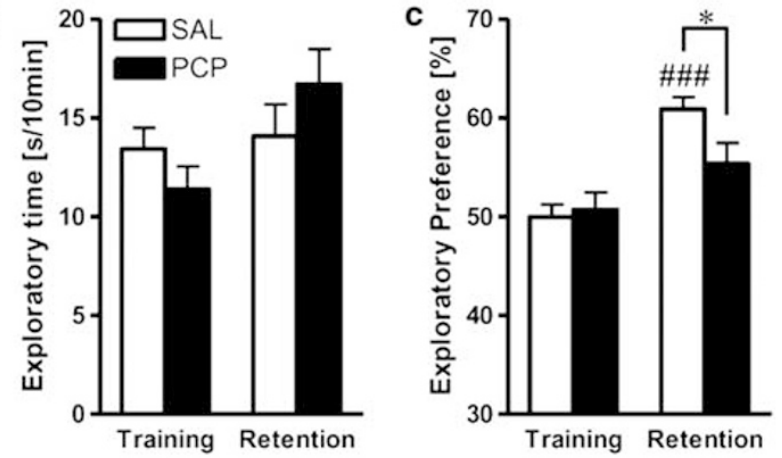

d

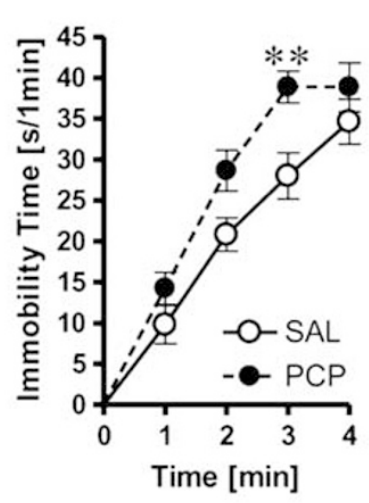

e

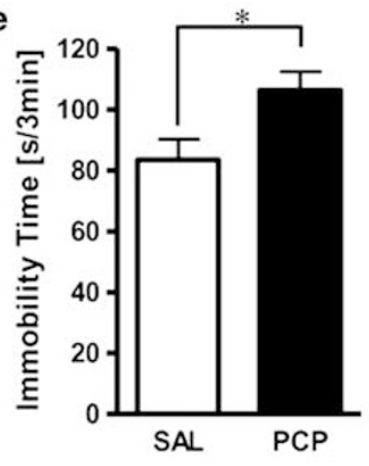

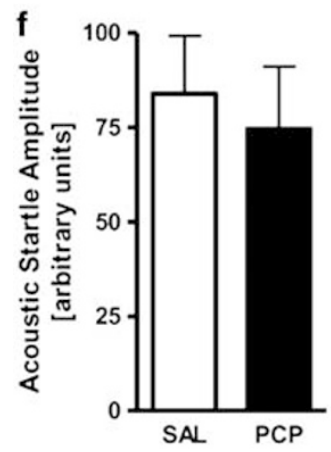

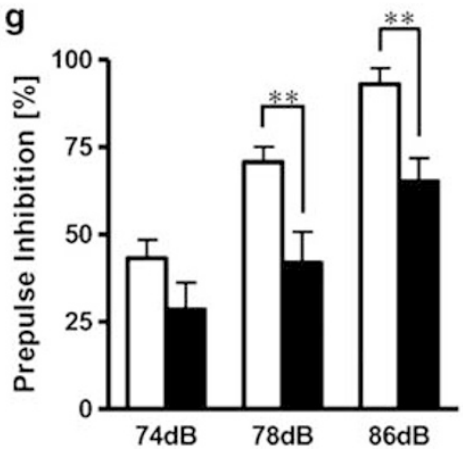

Figure 2 Prenatal PCP treatment induced behavioral deficits in adult. (a) Experimental schedule: mice were tested from P56. In the novel object recognition test, (b) exploratory time and (c) exploratory preference were determined. \#\#\#<0.001 compared with SAL-treated mice in the training session by paired $t$-test. ${ }^{*} p<0.05$ by Student's $t$-test. In the forced swimming test, (d) the time course (two-way ANOVA with repeated measures: $F_{\text {Interaction }(3,114)}=1.41 ; p>0.05, F_{\text {Time }(3,114)}=69.3 ; p<0.000 \mathrm{I}$, $\left.\mathrm{F}_{\text {Treatment }(1,114)}=6.97 ; p<0.05\right)$ and $(\mathrm{e})$ total scores of the immobility time were determined. ${ }^{*} p<0.05$, ** $p<0.0$ I by Student's $t$-test. In the PPI test, (f) acoustic startle amplitude without prepulse and (g) PPI (two-way ANOVA with repeated measures: $F_{\text {Interaction }(2.74)}=1.16 ; \quad p>0.05$, $\left.\mathrm{F}_{\text {Prepulse }(2.74)}=34.4 ; p<0.0001, \mathrm{~F}_{\text {Treatment }(1.74)}=11.65 ; p<0.01\right)$ were assessed. $* * 0<0.01$ by Student's $t$-test. Values are means \pm SEMs $(n=20)$. 


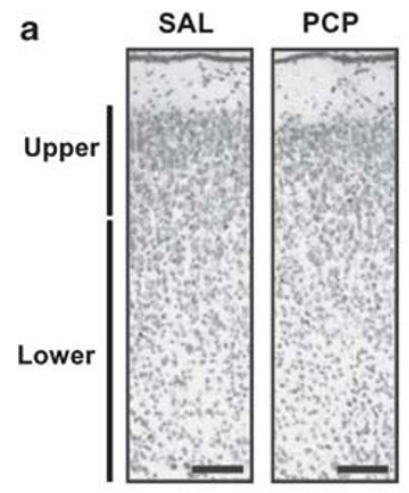

b

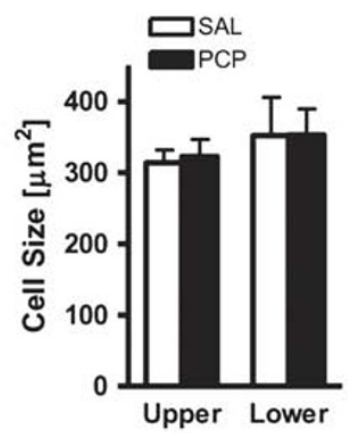

d

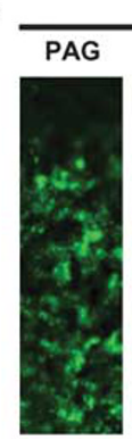

SAL

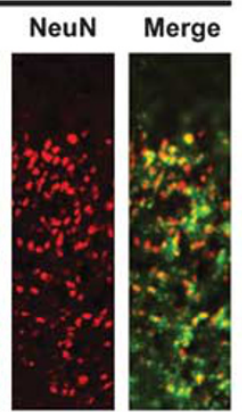

PCP

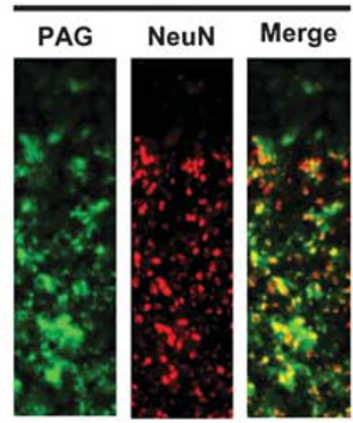

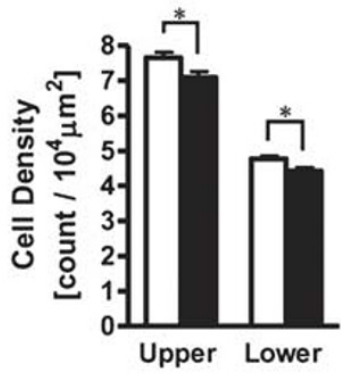

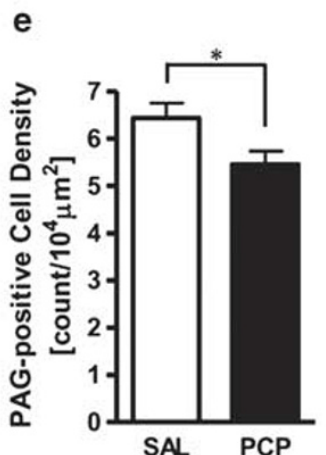

Figure 3 Prenatal PCP-treatment reduced the density of glutamatergic neurons in PFC. (a) Nissl-stained coronal sections of the PFC in SAL- and PCP-treated mice. Scale bar: $200 \mu \mathrm{m}$. Stereological analysis of (b) the size and (c) density of Nissl-stained cells in SAL- and PCP-treated mice ( $n=6$ ). (d) PAG-immunostained coronal sections of the PFC in PCP-and SAL-treated mice. Stereological analysis of (e) the density of PAG-stained cells was shown. Values are means \pm SEMs $(n=8)$. Scale bar: $200 \mu$ m. ${ }^{*} p<0.05$ by Student's $t$-test.

reported that neuronal migration is activated via the NMDA receptor (Komuro and Rakic, 1993). If neural migration was impaired by PCP treatment, the density of glutamatergic neurons in the PFC might be decreased, resulting from the ectopic distribution. To verify this possibility, we performed a migration assay using BrdU, by which newborn cells at the time of injection can be labeled, because it is incorporated into the newly synthesized DNA. On E12.5, E13.5, E14.5, or E15.5, pregnant mice were injected with BrdU $3 \mathrm{~h}$ after receiving PCP (Figure 4a). The $\mathrm{BrdU}^{+}$cells in PCP-treated mice on P7 were distributed normally with an inside-out pattern, and there were no significant changes compared with SAL-treated mice (Figure $4 \mathrm{~b}$ ). However, the density of $\mathrm{BrdU}^{+}$cells was decreased significantly in the early developmental stage (Figure 4c). This decrease was already evident at $30 \mathrm{~min}$ after the BrdU administration in PCP-treated mice (Figures $4 \mathrm{~d}$ and e). Moreover, another NMDA receptor antagonist, MK-801, induced a similar decrease in $\mathrm{BrdU}^{+}$cells. These results suggest that NMDA receptor antagonism might disturb the mitosis of neuronal progenitors.

\section{NMDA Receptor Antagonism Reduced the Density of Neuronal Progenitors in the VZ and SVZ}

Three main types of cortical neuronal progenitors have been identified throughout corticogenesis: radial glial cells, short neural precursors, and basal progenitor cells (Dehay and Kennedy, 2007). Both the short neural precursors and radial glia divide at the apical surface of the VZ and expresses a transcription factor, Pax6. The basal progenitor is the other major type of neuron-producing progenitor and is located in the SVZ, and expresses the transcription factor Tbr2. The SVZ starts to form on E13.5 in the mouse and expands significantly during late corticogenesis. To investigate the effect of NMDA receptor antagonism on neurogenesis, we checked these neural progenitors in the VZ and SVZ. The results showed that the number of $\mathrm{Pax}^{+}$neural progenitors on E13.5 was decreased in PCP-treated mice in comparison with SAL-treated mice, and $\mathrm{PCNA}^{+}$, a mitotic cell marker, cells were decreased also (Figures 5a, $c$ and d). Further, $\mathrm{Tbr}^{+}$basal progenitors were also decreased significantly in PCP-treated mice compared with SALtreated mice (Figures $5 \mathrm{~b}$ and e). These results suggest that prenatal NMDA antagonism might impair the proliferation of neural progenitors, resulting in the decrease in glutamatergic neurons.

\section{Prenatal PCP-Treatment Disrupted the Gene Expression Involved in Neurogenesis in Neural Progenitors}

To uncover the changes in gene expression in neuronal progenitors causing the impairment of proliferation by $\mathrm{PCP}$, we used a PCR array focusing on neurogenesis and neural stem cells. First, on E13.5, we cut out the VZ and SVZ, the areas containing neuronal progenitors, using LCM (Supplementary Figure S2). After the purification of mRNA from the tissue sections, we performed a comprehensive analysis of the expression of factors involved in neurogenesis and neuronal stem cells using the PCR array. 


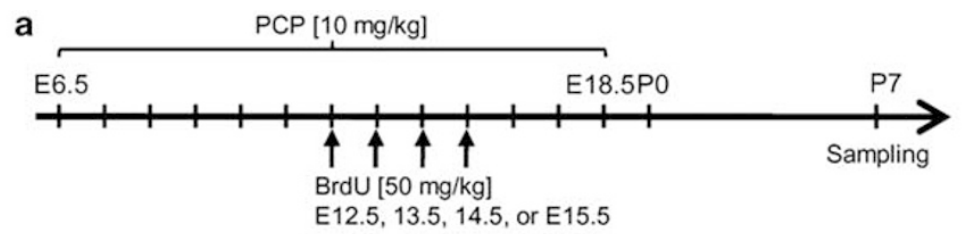

b
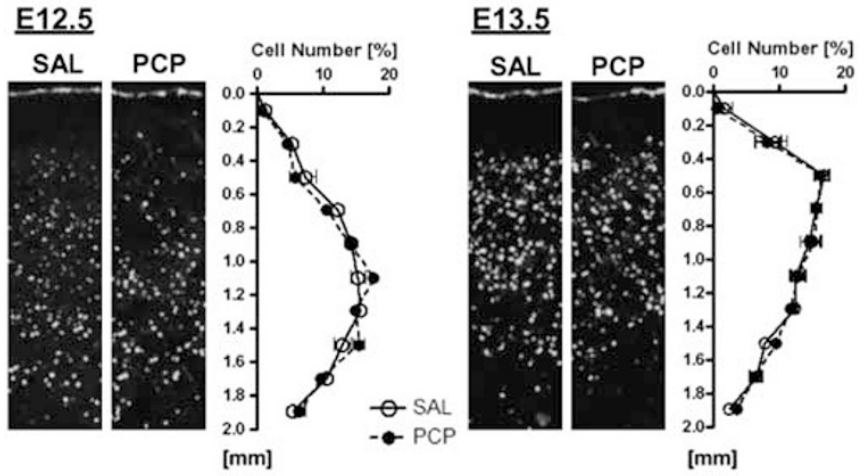

E14.5
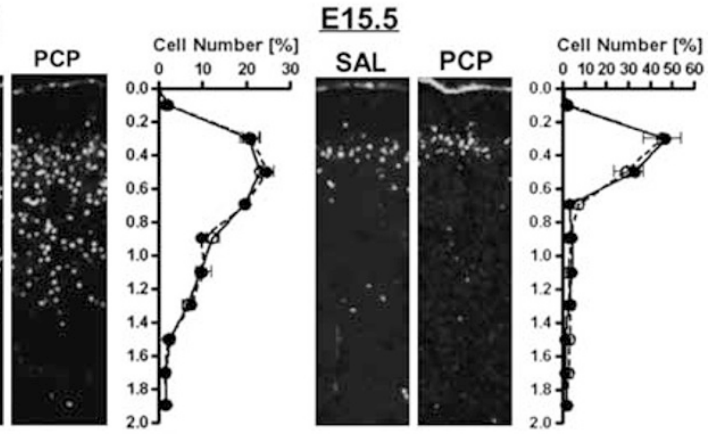

C

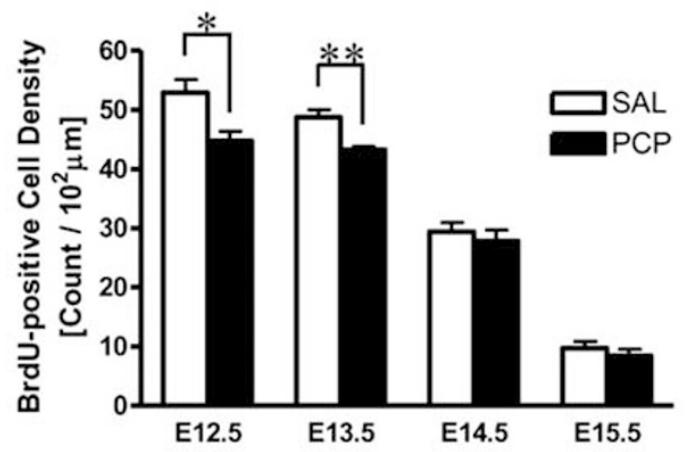

d

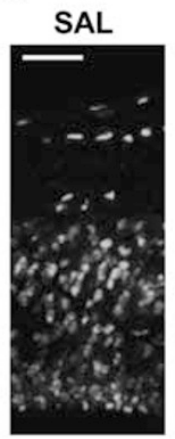

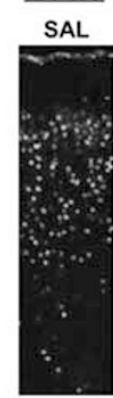

[mm]

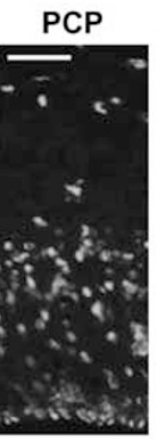

e

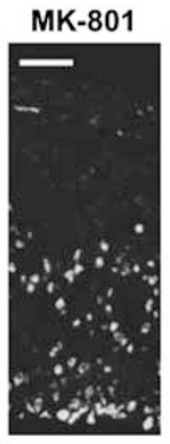

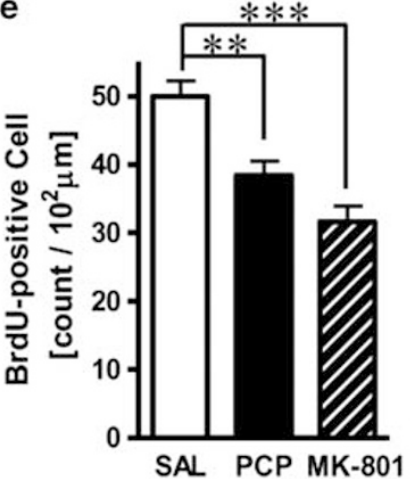

Figure 4 Prenatal PCP treatment affected the mitosis of neuronal progenitor but not neuronal migration. (a) Experimental schedule: the pregnant mice were given a single BrdU injection on EI 2.5, EI3.5, EI4.5, or EI 5.5, $3 \mathrm{~h}$ after the PCP/SAL administration to label mitotic progenitors in the embryonic brains. After the birth, the pups were fed for 7 days to examine cell migration. (b) Fluorescence images of BrdU ${ }^{+}$cells using the antibody and the percentage of $\mathrm{BrdU}^{+}$cells at each distance from the apical side are shown. (Two-way ANOVA with repeated measures: $E I 2.5$ ( $F_{\text {Interaction }(9,100)}=1.57$; $\left.p>0.05, F_{\text {Position }(9,100)}=86.4 ; p<0.0001, F_{\text {Treatment }(1,100)}<0.000 I ; p>0.05\right)$, El 3.5 ( $F_{\text {Interaction(9, 100) }}=0.47 ; p>0.05, F_{\text {Position(9, 100) }}=55.8 ; p<0.000 I$,

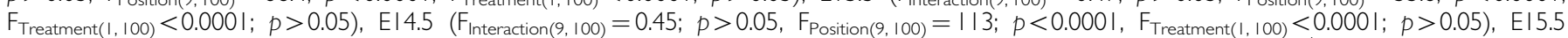
$\left(F_{\text {Interaction }(9,100)}=0.40 ; p>0.05, F_{\text {Position }(9,100)}=68.1 ; p<0.0001\right.$, $\left.\left.F_{\text {Treatment }(1,100)}=0.0001 ; p>0.05\right)\right)$. (c) The number of BrdU $U^{+}$cells per $100 \mu m$ in the tangential direction is shown $(n=6)$. ${ }^{*} p<0.05$, *** $p<0.01$ by Student's $t$-test. Furthermore, the PCP-, MK-80 I-, or SAL-treated pregnant mice were given a single $\mathrm{BrdU}$ injection $3 \mathrm{~h}$ before decapitation. (d) Fluorescence images of $\mathrm{BrdU}^{+}$cells in embryonic cortex are shown. Scale bar: I00 $\mu \mathrm{m}$. (e) Stereological analysis of the number of $\mathrm{BrdU}^{+}$cells per $100 \mu \mathrm{m}$ in the tangential direction in PCP-, MK-80I-, and SAL-treated mice is shown $(n=6)$. *** $<0.0 \mathrm{I}$, ***** $p<0.00$ I by Boferroni's test (one-way ANOVA: $F_{(2,21)}=17.0$ ). Values are means \pm SEMs.

Among genes whose $p$-value differed between the SAL- and PCP-treated groups by $<0.05$ and whose absolute value of change was larger than twofold, one gene was increased, and 16 genes decreased in expression in PCP-treated mice (Table 1). Two genes, Netrin1 (Ntn1) and Notch2, showed significant reductions in PCP-treated mice compared with SAL-treated mice. The result suggests that prenatal NMDA antagonism might disrupt the gene expression in neural progenitors, leading to the impairment of proliferation.

\section{DISCUSSION}

In this study, mice with prenatal exposure to PCP showed schizophrenia-like behavior in adulthood, such as recognition memory deficits and PPI impairments (Figure 2). These results are consistent with our previous study with PCP $(20 \mathrm{mg} / \mathrm{kg})$ (Lu et al, 2010), although the dose of PCP was lower $(10 \mathrm{mg} / \mathrm{kg})$ in this experiment. These behavioral deficits are recovered by treatment with a psycotropic drug such as clozapine. By crossing the placenta (Kaufman et al, 1983; Nicholas et al, 1982), PCP affects the developing fetal brain directly. Fico and Vanderwende (1988) found that PCP was rapidly transported into the fetal brain and disappeared in $8 \mathrm{~h}$ after maternal exposure during pregnancy. These findings suggest that prenatal PCP exposure could block NMDA receptors on the neuronal progenitors and/or newborn cells in the fetal brain, impairing neurodevelopment. As evidence of this, we showed that prenatal PCP-treatment decreased the body weight of the dams and newborn pups (Figure 1). The decrease in weight among the dams suggests impaired neurodevelopment. However, 

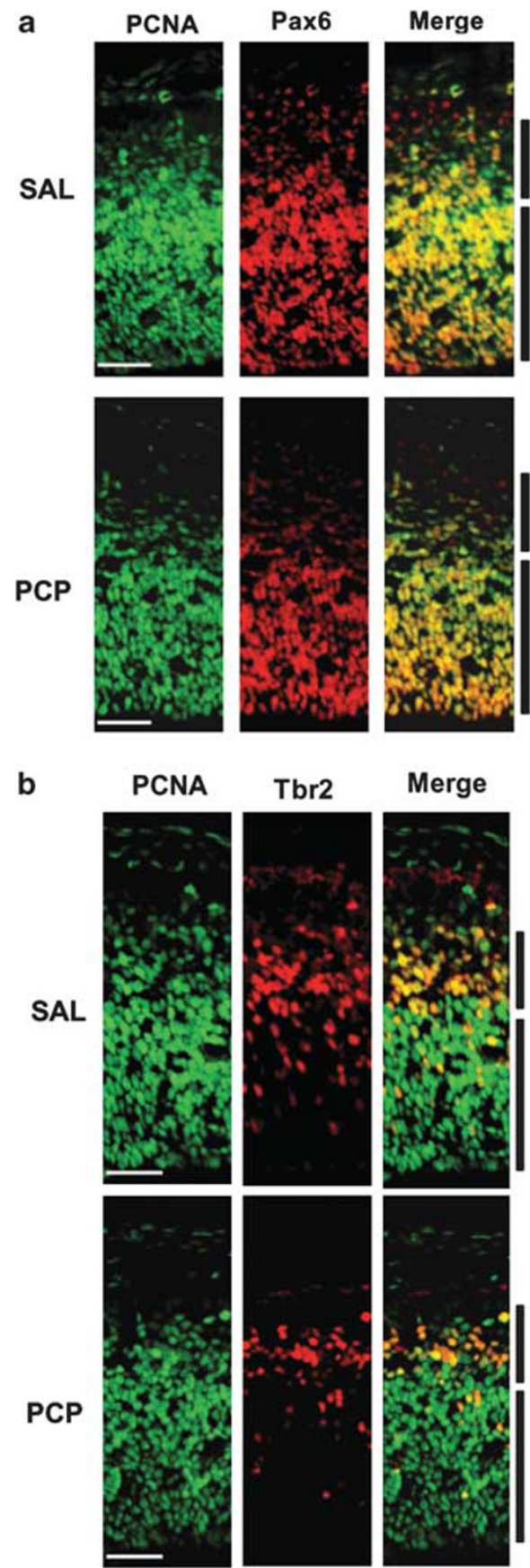

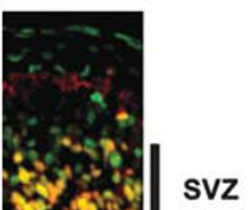

C
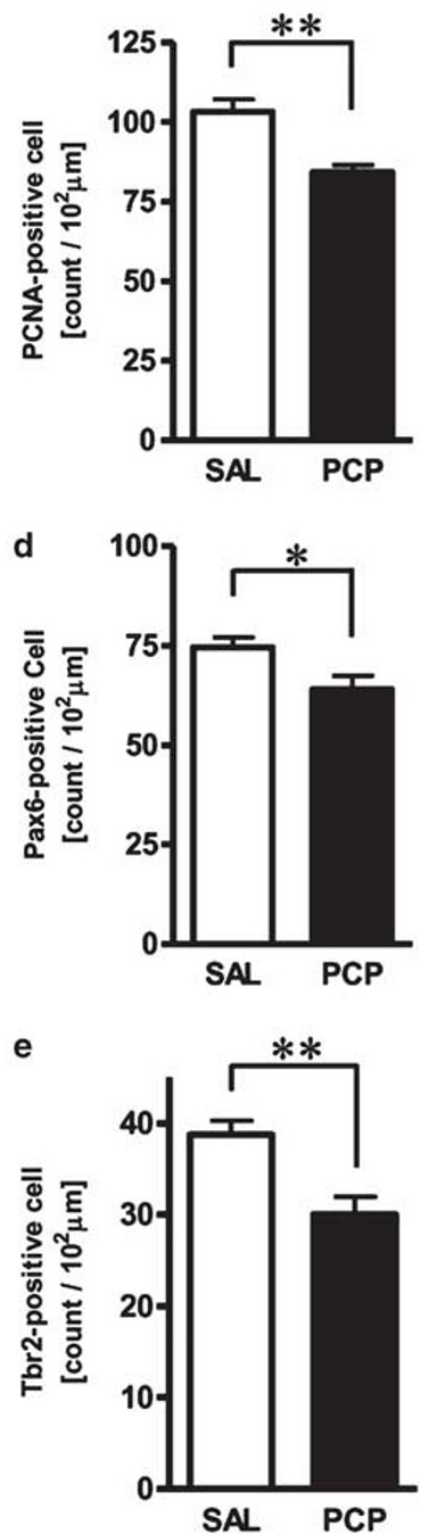

Figure 5 Prenatal PCP-treatment reduced the density of neuronal progenitors. On EI3.5, mice were decapitated $6 \mathrm{~h}$ after the PCP/SAL injection. The pups were analyzed by immunohistochemical analysis using $\alpha$-PCNA as a marker of proliferation, $\alpha$-Pax 6 antibody as a marker for neuronal progenitors in VZ, and $\alpha$-Tbr2 antibody as a marker for basal progenitors in SVZ. Fluorescence images of PCNA-, (a) Pax6-, and (b) Tbr2-positive cells in embryonic cortex are shown. Scale bar: $100 \mu \mathrm{m}$. The density of (c) PCNA-, (d) Pax6-, and (e) Tbr2-positive cells per $100 \mu \mathrm{m}$ in the tangential direction is shown. Data are means \pm SEMs $(n=6)$. * $p<0.05, * * x<0.0$ I by Student's $t$-test.

because PCP administration was reported to reduce body weight in non-pregnant adult mice (Koseki et al, 2011), the decrease must result from a reduction in not only the weight of the pups but also that of the dam itself. On the other hand, the decrease in the weight of newborn pups on P1 strongly indicates that prenatal PCP treatment impaired neurodevelopment. Moreover, the difference in weight decreased with growth to the point that there was no difference on P28 and 56, suggesting that prenatal treatment with PCP has little impact on development after birth. These results are consistent with our previous study ( $\mathrm{Lu}$ et al, 2010).

We found that the density, but not size, of glutamatergic neurons, was decreased in the PFC of the PCP-treated mice on P56 compared with SAL-treated mice (Figure 3). These findings might be consistent with the report that the CNS structures of NR1 knock-out mice are somewhat smaller than normal, although no region is defected obviously 
Table I List of Gene Whose Expression in Progenitor Cells was Altered by PCP

\begin{tabular}{|c|c|c|c|c|c|c|}
\hline & Gene name & Symbol & $\begin{array}{l}\text { Gene } \\
\text { number }\end{array}$ & $\begin{array}{l}\text { Fold difference } \\
\text { PCP/SAL }\end{array}$ & $\begin{array}{c}\text { t-test } \\
p \text {-value }\end{array}$ & $\begin{array}{c}\text { Fold up- or } \\
\text { down- PCP/SAL }\end{array}$ \\
\hline \multirow[t]{10}{*}{ Decrease } & Disheveled 3, dsh homolog (Drosophila) & Dvl3 & NM_007889 & 0.320 & 0.335 & -3.12 \\
\hline & Filamin, alpha & Flna & NM_0I0227 & 0.370 & 0.494 & -2.70 \\
\hline & Guanine nucleotide-binding protein, alpha o & Gnaol & NM_0I0308 & 0.148 & 0.294 & -6.76 \\
\hline & Nuclear receptor coactivator 6 & Ncoa6 & NM_019825 & 0.289 & 0.203 & -3.46 \\
\hline & Notch gene homolog 2 (Drosophila) & Notch2 & NM_0I0928 & 0.546 & $0.047 *$ & -1.83 \\
\hline & Neuronal pentraxin I & Nptxl & NM_008730 & 0.440 & 0.333 & -2.27 \\
\hline & Neuron-glia-CAM-related cell adhesion molecule & Nrcam & NM_I 76930 & 0.255 & 0.418 & -3.92 \\
\hline & Netrin I & Ntn l & NM_008744 & 0.257 & $0.014 *$ & -3.89 \\
\hline & SRY-box containing gene 3 & Sox3 & NM_009237 & 0.475 & 0.462 & -2.11 \\
\hline & $\begin{array}{l}\text { Tyrosine 3-monooxygenase/tryptophan 5-monooxygenase } \\
\text { activation protein, eta polypeptide }\end{array}$ & Ywhah & NM_0II738 & 0.382 & 0.658 & -2.62 \\
\hline Increase & Paired box gene 6 & Pax6 & NM_0I3627 & 2.666 & 0.573 & 2.67 \\
\hline
\end{tabular}

*p<0.05: Student's t-test $(n=5)$.

affected (Forrest et al, 1994). This reduction in the density of glutamatergic neurons would result in glutamatergic hypofunction in the PFC. In fact, we have already reported that active phosphorylated NR1, an essential subunit of the NMDA receptor, was decreased (Lu et al, 2010), and the basal extracellular glutamate level and high $\mathrm{K}^{+}$-evoked glutamate release were also reduced in the PFC of PCPtreated mice ( $\mathrm{Lu}$ et al, 2011). Furthermore, we have confirmed that the release of glutamate was decreased in the PFC of PCP-treated mice, and some abnormal behaviors were improved by treatment with D-serine, a co-agonist of the NMDA receptor, and a glutamate aspartate transporter inhibitor (Lu et al, 2010). These results suggest that a blockade of NMDA receptors by PCP might prevent glutamatergic neurodevelopment, resulting in hypoglutamatergic function in the PFC.

Although we focused on glutamatergic neurons in this study, it was reported that prenatal NMDA receptor blockade affects the development of other types of neuron such as the GABAergic neuron. For example, prenatal exposure (E15-E18) to MK-801 reduced the density of parvalbumin-positive GABA neurons in the rat medial PFC on P63 and enhanced PCP-induced hyperlocomotion (Abekawa et al, 2007). This finding suggests that prenatal treatment with PCP might impair the development of not only glutamatergic but also GABAergic neurons, leading to GABAergic dysfunction in the PFC. Further experiments focusing on GABAergic neurons should be performed using our model.

During the development of the mammalian cortex, neuronal progenitor cells generate neurons through asymmetric and symmetric divisions. In the early stages, $\mathrm{Pax6}^{+}{ }^{-}$ neural progenitors undergo symmetric, proliferative divisions, each of which generates two progenitor cells in the VZ (Rakic, 1995; McConnell, 1995). These divisions are followed by many asymmetric divisions, each of which generates a daughter progenitor plus a more differentiated cell such as a $\mathrm{Tbr} 2^{+}$ basal progenitor and neuron. The remaining neuronal progenitors in the VZ and basal progenitors in the SVZ typically undergo symmetric, differentiating divisions, each of which generates two neurons (Götz and Huttner, 2005; Hevner, 2006). These neurons migrate radially to the proper site, resulting in the formation of a layered structure with an inside-out pattern. We found that prenatal PCP treatment reduced the density of $\mathrm{BrdU}^{+}$cells in early developmental stages (Figure 4), in which mainly symmetric, proliferative divisions of neuronal progenitor were underway. Actually, we confirmed a reduction in both types of neuronal progenitor cells in the VZ and SVZ (Figure 5). Many reports have said that the NMDA receptor has an important role in the proliferation of neuronal progenitors. Glutamate stimulation via NMDA receptors significantly increased the proliferation of human neural progenitor cells derived from the fetal frontal cortex (Suzuki et al, 2006). Neurospheres derived from the embryonic rat brain express the NR1 and NR2B subunits of the NMDA receptor and show reductions in diameter and number (Mochizuki et al, 2007). Furthermore, the activation of NMDA receptors increases the proliferation of neuronal progenitors in the developing hippocampus (Joo et al, 2007) and striatum (Luk et al, 2003) as well. These findings are consistent with the present results. Further, it is easy to assume that the reduction in progenitors causes the low density of glutamatergic neurons, because these progenitors mainly produce glutamatergic neurons.

Previous reports have shown that the stimulation of NMDA receptors promotes the radial migration of neurons 
(Komuro and Rakic, 1993). However, in this study, prenatal PCP treatment did not affect the migration. One explanation is that the half-life of PCP in brain tissue is very short: the concentration of PCP in rat fetal brain peaked $30 \mathrm{~min}$ after its injection into pregnant dams, and the half-life of PCP on E15 and E18 in brain tissue was 126 and $27 \mathrm{~min}$, respectively (Fico and Vanderwende, 1988). The neuronal migration might be impaired temporarily by $\mathrm{PCP}$, but soon return to normal as the PCP in fetal brain disappears. However, prenatal PCP treatment might have a critical impact on neuronal progenitors because of repeated exposure.

We showed that prenatal PCP-treatment disrupted the gene expression of Notch2 and Ntn1, in neural progenitors (Table 1). The Notch pathway is involved in a wide array of cell fate decisions during development (Louvi and Artavanis-Tsakonas, 2006). Mammalian Notch proteins appear to have an important role in preventing cell differentiation in a variety of cell lineages. Notably, in the developing cerebellar cortex, Notch2 signaling in granule neuron precursors has been reported to inhibit differentiation into neurons and maintain precursor proliferation (Solecki et al, 2001). This suggests that decreased Notch2 expression in progenitor cells of PCP-treated mice might prevent the proliferation of neuronal progenitors in early developmental stages, leading to the low density. Further, $\mathrm{Ntn} 1$ is a diffusible protein, which provides informational cues for several cellular functions, including cell adhesion, migration, axon guidance, proliferation, differentiation, and cell survival (Cirulli and Yebra, 2007). A recent report has demonstrated that one Ntn1 receptor, Neogenin, is expressed in neuronal progenitors including radial glia during neurogenesis in the forebrain. The Neogenin-positive progenitor displays higher proliferative and neurogenic potential than its negative counterpart (Fitzgerald et al, 2007), suggesting that Ntn1 signaling might activate neurogenesis. As well as Notch2, the decreased Ntn1 expression might bring about the impairment of proliferation. Furthermore, both Notch2 and Ntn1 signaling has been reported to be involved in apoptotic cell death. Apoptosis is enhanced in both Notch2 and Ntn1 mutant fetal brain (Hamada et al, 1999; Llambi et al, 2001). Interestingly, it also occurred in the fetal brains of prenatal PCP-treated rats (Ikonomidou et al, 1999). Apoptosis via the decrease in Notch 2 and Ntn 1 might be one of the causes of the low density of neuronal progenitors.

In this study, prenatal PCP treatment affected the expression of only two genes in the neuronal progenitors, although it induced very severe behavioral deficits in adulthood. The expression of more genes is likely to be changed by the treatment. However, the PCR array system used in this study can evaluate the expression of only 84 genes. Moreover, genes having important functions other than in neurogenesis were not checked. To evaluate more exhaustively the effect of NMDA receptor antagonism on gene expression, PCR arrays and micro arrays with a broader range would be required.

In conclusion, as a mechanism by which a prenatal NMDA receptor blockade causes long-lasting behavioral changes, we demonstrated that prenatal NMDA receptor antagonism impaired the proliferation of neuronal progenitors through aberrant gene expression, and consequently decreased the density of glutamatergic neurons in the PFC, resulting in glutamatergic hypofunction. These findings lead us to speculate that NMDA receptors regulate the proliferation and maturation of progenitor cells in the $\mathrm{VZ}$ during neurodevelopment via the regulation of gene expression.

\section{ACKNOWLEDGEMENTS}

We thank Dr Furukawa $\mathrm{H}$ for synthesizing PCP. This study was supported by Grants-in-aid for Scientific Research (A) (22248033), Scientific Research (B) (20390073) (21390045) and Exploratory Research from the JSPS (19659017) (22659213); by the 'Academic Frontier' Project for Private Universities (2007-2011) from the Ministry of Education, Culture, Sports, Science and Technology of Japan (MEXT); by Regional Joint Research Program supported by grants to Private Universities to Cover Current Expenses from MEXT; by Research on Regulatory Science of Pharmaceuticals and Medical Devices from the Ministry of Health, Labour and Welfare, Japan (MHLW); by Research on Risk of Chemical Substances, Health and Labour Science Research Grants supported by MHLW; and by the joint research project under the Japan-Korea basic scientific cooperation program (JSPS).

\section{DISCLOSURE}

The authors declare no conflict of interest.

\section{REFERENCES}

Abekawa T, Ito K, Nakagawa S, Koyama T (2007). Prenatal exposure to an NMDA receptor antagonist, MK-801 reduces density of parvalbumin-immunoreactive GABAergic neurons in the medial prefrontal cortex and enhances phencyclidineinduced hyperlocomotion but not behavioral sensitization to methamphetamine in postpubertal rats. Psychopharmacology (Berlin) 192: 303-316.

Asahi M, Hoshimaru M, Hojo M, Matsuura N, Kikuchi H, Hashimoto $N$ (1998). Induction of the N-methyl-D-aspartate receptor subunit 1 in the immortalized neuronal progenitor cell line HC2S2 during differentiation into neurons. J Neurosci Res 52: 699-708.

Brazel CY, Nuñez JL, Yang Z, Levison SW (2005). Glutamate enhances survival and proliferation of neural progenitors derived from the subventricular zone. Neuroscience 131: 55-65.

Cirulli V, Yebra M (2007). Netrins: beyond the brain. Nat Rev Mol Cell Biol 8: 296-306.

Dehay C, Kennedy H (2007). Cell-cycle control and cortical development. Nat Rev Neurosci 8: 438-450.

Fico TA, Vanderwende C (1988). Phencyclidine during pregnancy: fetal brain levels and neurobehavioral effects. Neurotoxicol Teratol 10: 349-354.

Fitzgerald DP, Bradford D, Cooper HM (2007). Neogenin is expressed on neurogenic and gliogenic progenitors in the embryonic and adult central nervous system. Gene Expr Patterns 7: 784-792.

Forrest D, Yuzaki M, Soares HD, Ng L, Luk DC, Sheng M et al (1994). Targeted disruption of NMDA receptor 1 gene abolishes NMDA response and results in neonatal death. Neuron 13: 325-338.

Götz M, Huttner WB (2005). The cell biology of neurogenesis. Nat Rev Mol Cell Biol 6: 777-788.

Hamada Y, Kadokawa Y, Okabe M, Ikawa M, Coleman JR, Tsujimoto $Y$ (1999). Mutation in ankyrin repeats of the mouse 
Notch2 gene induces early embryonic lethality. Development 126: 3415-3424.

Heins N, Malatesta P, Cecconi F, Nakafuku M, Tucker KL, Hack MA et al (2002). Glial cells generate neurons: the role of the transcription factor Pax6. Nat Neurosci 5: 308-315.

Hevner RF (2006). From radial glia to pyramidal-projection neuron: transcription factor cascades in cerebral cortex development. Mol Neurobiol 33: 33-50.

Ikonomidou C, Bosch F, Miksa M, Bittigau P, Vöckler J, Dikranian $\mathrm{K}$ et al (1999). Blockade of NMDA receptors and apoptotic neurodegeneration in the developing brain. Science 283: 70-74.

Joo JY, Kim BW, Lee JS, Park JY, Kim S, Yun YJ et al (2007). Activation of NMDA receptors increases proliferation and differentiation of hippocampal neural progenitor cells. J Cell Sci 120: 1358-1370.

Kaneko T, Mizuno N (1994). Glutamate-synthesizing enzymes in GABAergic neurons of the neocortex: a double immunofluorescence study in the rat. Neuroscience 61: 839-849.

Kaufman KR, Petrucha RA, Pitts Jr FN, Weekes ME (1983). PCP in amniotic fluid and breast milk: case report. J Clin Psychiatry 44: 269-270.

Komuro H, Rakic P (1993). Modulation of neuronal migration by NMDA receptors. Science 260: 95-97.

Koseki T, Mouri A, Mamiya T, Aoyama Y, Toriumi K, Suzuki S et al (2011). Exposure to enriched environments during adolescence prevents abnormal behaviors associated with histone deacetylation in phencyclidine-treated mice. Int J Neuropsychopharmacol (in press).

Lewis DA, Glantz LA, Pierri JN, Sweet RA (2003). Altered cortical glutamate neurotransmission in schizophrenia: evidence from morphological studies of pyramidal neurons. Ann NY Acad Sci 1003: 102-112.

Llambi F, Causeret F, Bloch-Gallego E, Mehlen P (2001). Netrin-1 acts as a survival factor via its receptors UNC5H and DCC. EMBO J 20: 2715-2722.

López T, López-Colomé AM, Ortega A (1997). NMDA receptors in cultured radial glia. FEBS Lett 405: 245-248.

Louvi A, Artavanis-Tsakonas S (2006). Notch signalling in vertebrate neural development. Nat Rev Neurosci 7: 93-102.

Lu L, Mamiya T, Lu P, Toriumi K, Mouri A, Hiramatsu M et al (2010). Prenatal exposure to phencyclidine produces abnormal behavior and NMDA receptor expression in postpubertal mice. Int J Neuropsychopharmacol 13: 877-889.

Lu L, Mamiya T, Lu P, Toriumi K, Mouri A, Hiramatsu M et al (2011). Prenatal exposure to PCP produces behavioral deficits accompanied by the overexpression of GLAST in the prefrontal cortex of postpubertal mice. Behav Brain Res 220: 132-139.
Luk KC, Kennedy TE, Sadikot AF (2003). Glutamate promotes proliferation of striatal neuronal progenitors by an NMDA receptor-mediated mechanism. J Neurosci 23: 2239-2250.

Maddox VH, Godefroi EF, Parcell RF (1965). The synthesis of phencyclidine and other 1-arylcyclohexylamines. J Med Chem 8: 230-235.

McConnell SK (1995). Constructing the cerebral cortex: neurogenesis and fate determination. Neuron 15: 761-768.

Mochizuki N, Takagi N, Kurokawa K, Kawai T, Besshoh S, Tanonaka K et al (2007). Effect of NMDA receptor antagonist on proliferation of neurospheres from embryonic brain. Neurosci Lett 417: 143-148.

Mouri A, Noda Y, Hara H, Mizoguchi H, Tabira T, Nabeshima T (2007). Oral vaccination with a viral vector containing Abeta cDNA attenuates age-related Abeta accumulation and memory deficits without causing inflammation in a mouse Alzheimer model. FASEB J 21: 2135-2148.

Murai R, Noda Y, Matsui K, Kamei H, Mouri A, Matsuba K et al (2007). Hypofunctional glutamatergic neurotransmission in the prefrontal cortex is involved in the emotional deficit induced by repeated treatment with phencyclidine in mice: implications for abnormalities of glutamate release and NMDA-CaMKII signaling. Behav Brain Res 180: 152-160.

Nicholas JM, Lipshitz J, Schreiber EC (1982). Phencyclidine: its transfer across the placenta as well as into breast milk. Am J Obstet Gynecol 143: 143-146.

Pfaffl MW (2001). A new mathematical model for relative quantification in real-time RT-PCR. Nucleic Acids Res 29: e45.

Rakic P (1995). A small step for the cell, a giant leap for mankind: a hypothesis of neocortical expansion during evolution. Trends Neurosci 18: 383-388.

Sah DW, Ray J, Gage FH (1997). Regulation of voltage- and ligandgated currents in rat hippocampal progenitor cells in vitro. J Neurobiol 32: 95-110.

Solecki DJ, Liu XL, Tomoda T, Fang Y, Hatten ME (2001). Activated Notch2 signaling inhibits differentiation of cerebellar granule neuron precursors by maintaining proliferation. Neuron 31: 557-568.

Suzuki M, Nelson AD, Eickstaedt JB, Wallace K, Wright LS, Svendsen CN (2006). Glutamate enhances proliferation and neurogenesis in human neural progenitor cell cultures derived from the fetal cortex. Eur J Neurosci 24: 645-653.

Wang C, McInnis J, Ross-Sanchez M, Shinnick-Gallagher P, Wiley JL, Johnson KM (2001). Long-term behavioral and neurodegenerative effects of perinatal phencyclidine administration: implications for schizophrenia. Neuroscience 107: 535-550.

Supplementary Information accompanies the paper on the Neuropsychopharmacology website (http://www.nature.com/npp)

Neuropsychopharmacology 